\title{
Ruptured Feeder Aneurysm Associated with Cerebellar Arteriovenous Malformation
}

\author{
Shushi Kominami ${ }^{1,2}$, Akira Watanabe ${ }^{1,2}$, Shiro Kobayashi ${ }^{1,2}$ and Akira Teramoto ${ }^{1}$ \\ ${ }^{1}$ Department of Neurosurgery, Graduate School of Medicine, Nippon Medical School \\ ${ }^{2}$ Department of Neurosurgery, Nippon Medical School Chiba Hokuso Hospital
}

\begin{abstract}
We report 2 cases of ruptured aneurysms of the posterior inferior cerebellar artery associated with an arteriovenous malformation (AVM). The aneurysm and AVM were simultaneously embolized with n-butyl cyanoacrylate. In one case both the aneurysm and the AVM were totally obliterated; in the other case the AVM was subsequently treated with radiosurgery. In both cases the aneurysms were successfully occluded. The effectiveness and limitations of this treatment are discussed.
\end{abstract}

(J Nippon Med Sch 2012; 79: 296-300)

Key words: arteriovenous malformation, cerebral aneurysm, embolization, n-butyl cyanoacrylate

\section{Introduction}

Cerebral arteriovenous malformations (AVMs) are sometimes associated with cerebral aneurysms of the feeding artery. When these aneurysms rupture, they become the primary target of treatment; surgical clipping has often been the treatment of choice $^{1.6 .7}$. Endovascular coil embolization is another treatment option ${ }^{3.5}$. We have treated 2 cases of feeder aneurysms associated with AVMs by means of embolization with n-butyl cyanoacrylate (NBCA).

\section{Case Reports}

\section{Case 1}

A 63-year-old woman with severe headache reported to a local hospital on foot 3 days after onset. Computed tomography (CT) of the head revealed a small intracerebellar hematoma in the vermis and an intraventricular hemorrhage in the fourth ventricle and posterior horn of the left lateral ventricle (Fig. 1). The patient was admitted and treated conservatively for hypertensive intracerebellar hemorrhage. Three weeks after onset she was referred to us for follow-up. She was free of symptoms except for mild headache. Magnetic resonance angiography revealed an aneurysm in the posterior fossa; digital subtraction angiography (DSA) showed an AVM on the posterior surface of the cerebellar vermis supplied by the bilateral posterior inferior cerebellar arteries (PICAs) and the left superior cerebellar artery (SCA) (Fig. 2). An aneurysm on the superior retrotonsillar segment of the left PICA was also demonstrated; no aneurysm neck was visualized. A microcatheter was

Correspondence to Shushi Kominami, MD, PhD, Department of Neurosurgery, Nippon Medical School Chiba Hokuso Hospital, 1715 Kamagari, Inzai, Chiba 270-1694, Japan

E-mail: shushi@nms.ac.jp

Journal Website (http://www.nms.ac.jp/jnms/) 


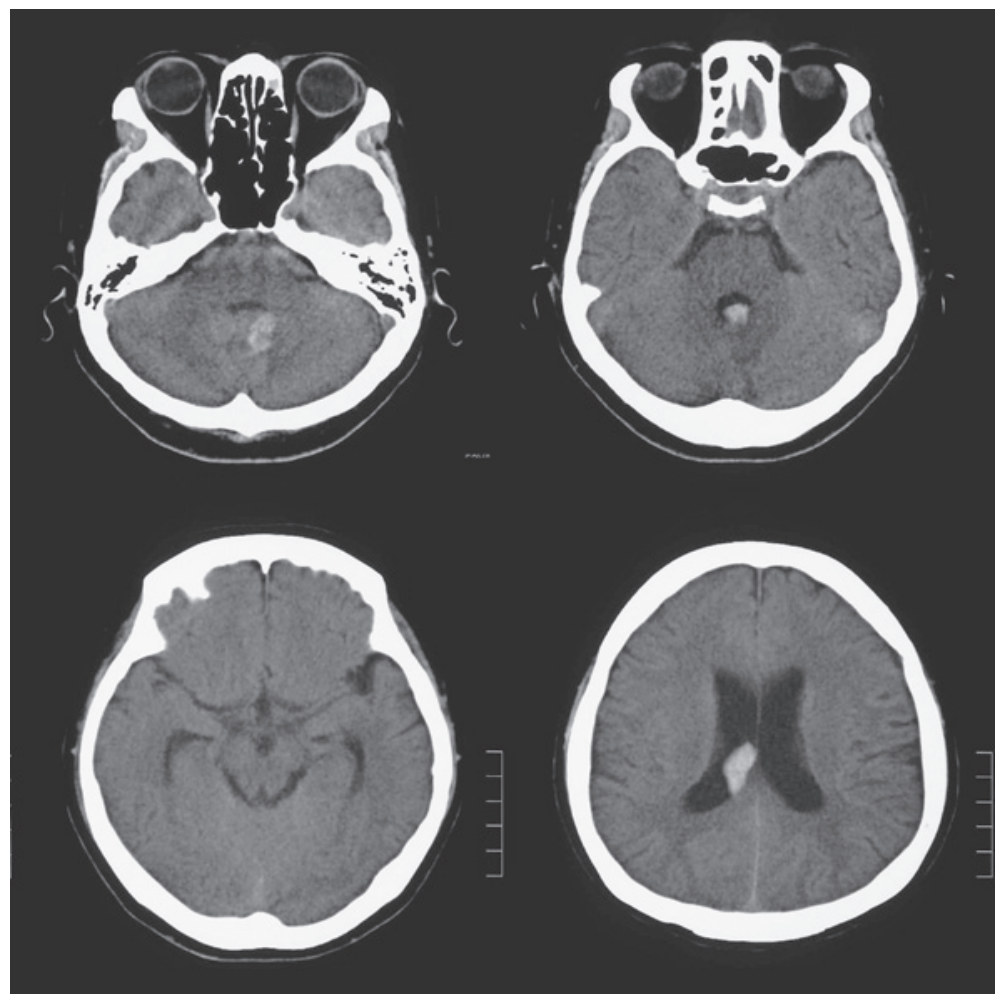

Fig. 1 The initial head CT scan showed intracerebellar and intraventricular but no obvious subarachnoid hemorrhage.

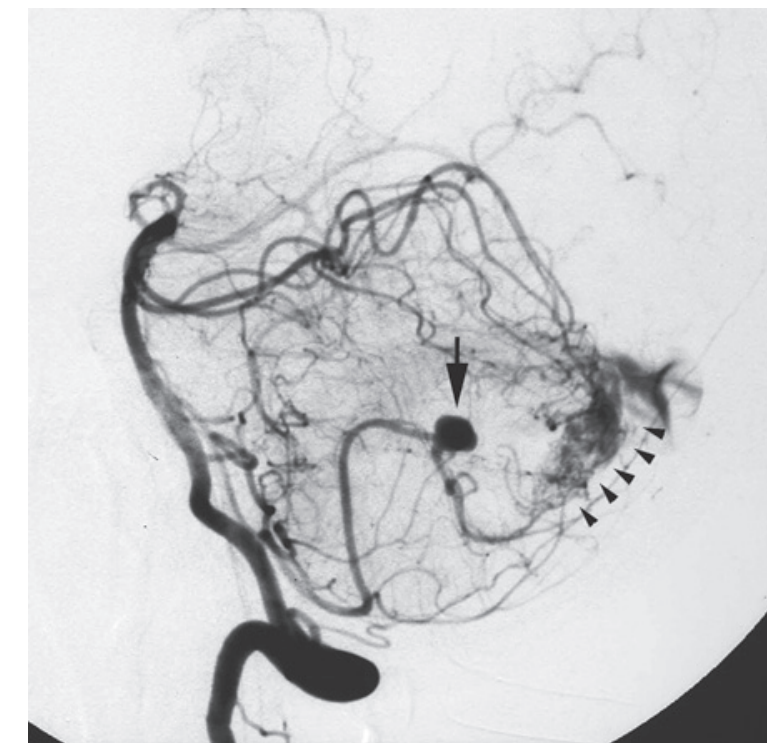

Fig. 2 Left vertebral angiogram showing a saccular aneurysm (arrow) at the superior retrotonsillar segment of the left PICA and an AVM (arrowheads) supplied by the PICA and SCA.

introduced into the left PICA, and superselective angiography showed that the aneurysm had arisen on the artery feeding the AVM. On the basis on its distribution observed on the $\mathrm{CT}$, we attributed the

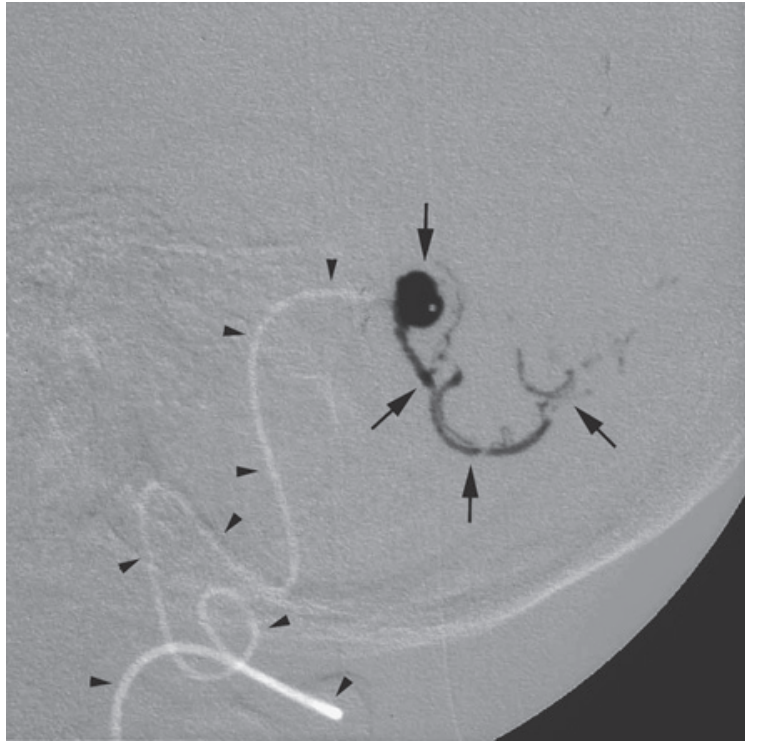

Fig. 3 DSA performed at the end of glue injection demonstrates the glue cast (arrows) and the retrieved catheter (arrowheads). Note that the glue filled the aneurysm and part of the AVM.

hemorrhage to the aneurysm rather than to the AVM.

We chose to treat the aneurysm and the AVM in a single procedure. The aneurysm was embolized 


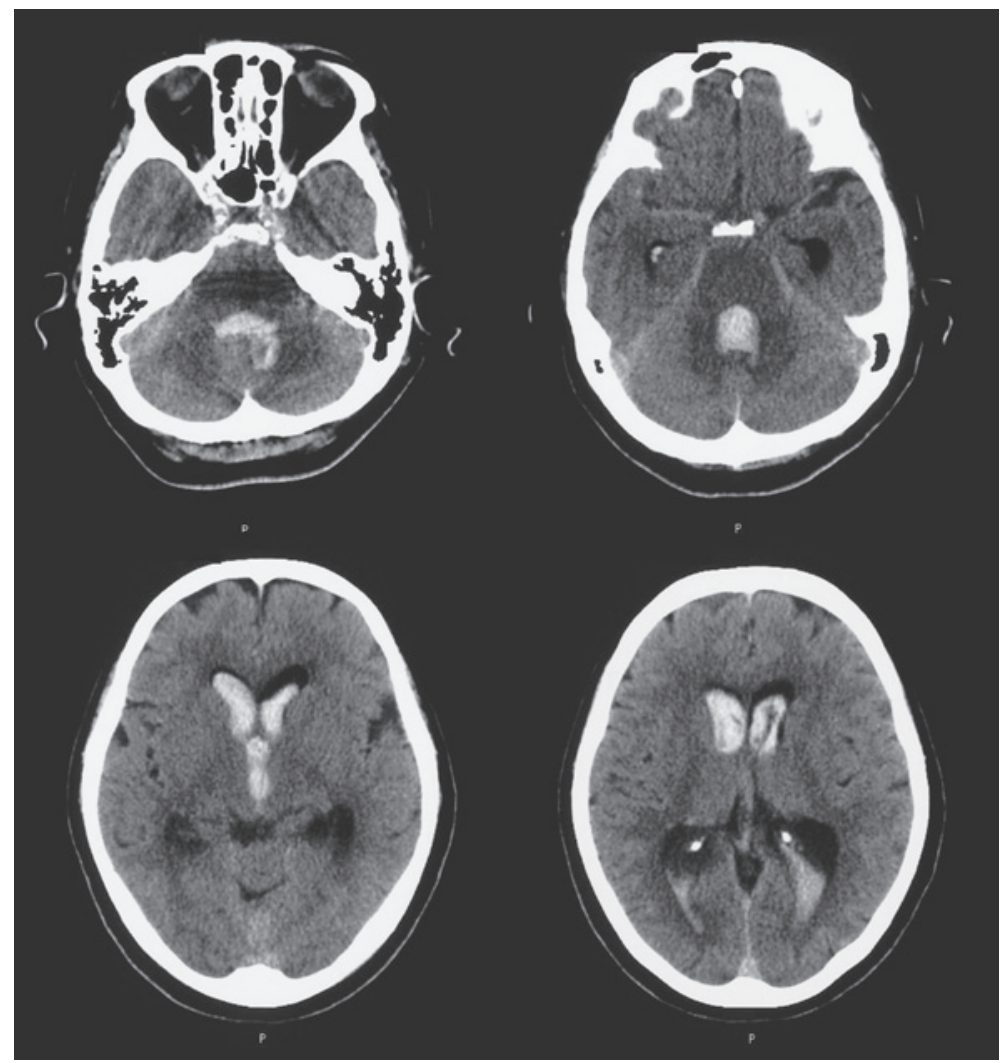

Fig. 4 Head CT scan obtained at admission shows intraventricular hemorrhage and dilated ventricles.

with a NBCA glue via a microcatheter advanced into the aneurysm. The glue was injected until it had penetrated the AVM (Fig. 3). The AVM was embolized with an additional embolization treatment through the right PICA. The patient was given heparin after embolization to avoid retrograde thrombosis of the PICA. Two hours after embolization the patient complained of worsening headache; CT scan showed new bleeding in the cerebellar vermis. We immediately neutralized the delivered heparin with protamine, and no further bleeding occurred. She exhibited no neurological deficits throughout the postoperative course. Followup angiography performed 3 months after treatment confirmed total obliteration of the aneurysm. The small residual AVM supplied from the left SCA was addressed by radiosurgery.

\section{Case 2}

A 61-year-old woman was admitted with suddenonset severe headache; intraventricular hemorrhage was observed in all ventricles (Fig. 4). She was

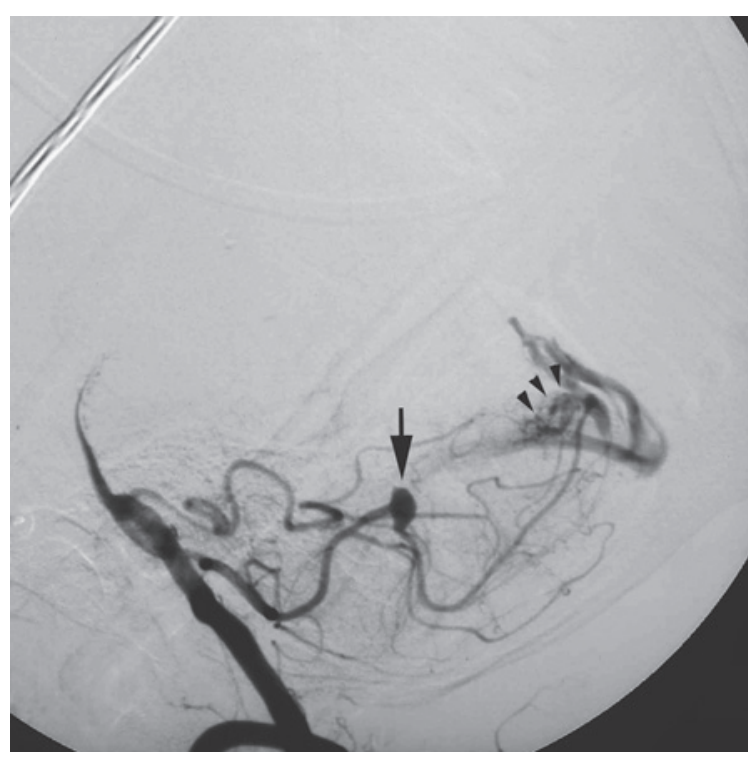

Fig. 5 Left vertebral angiogram demonstrates a saccular aneurysm (arrow) at the superior retrotonsillar segment of the left PICA and an AVM (arrowheads) supplied by the PICA.

neurologically intact on admission but her condition deteriorated gradually owing to obstructive 


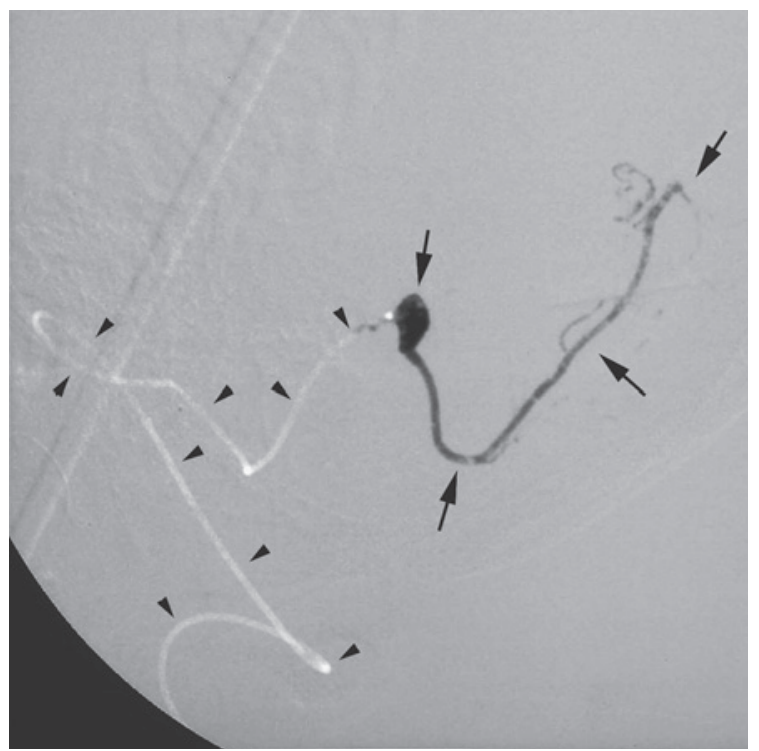

Fig. 6 DSA performed at the end of glue injection shows the glue cast (arrows) and the retrieved catheter (arrowheads). The aneurysm, a part of the AVM, and a part of the PICA proximal to the aneurysm are filled with glue.

hydrocephalus. External ventricular drainage resulted in her recovery. DSA revealed an aneurysm without a neck at the superior retrotonsillar segment of the left PICA and a small AVM on the posterior surface of the vermis (Fig. 5). The AVM was supplied by the left PICA and left SCA.

On the day of admission, we navigated a microcatheter into the left PICA just proximal to the aneurysm. The aneurysm and AVM were totally obliterated by a single injection of glue (Fig. 6). The ventricular drainage tube was removed 3 days later. She recovered fully except for transient truncal ataxia, which resolved within 3 months. Diffusionweighted magnetic resonance imaging showed a small infarction in the lower part of the cerebellar vermis. Follow-up angiography performed 3 months after treatment confirmed total obliteration of the aneurysm and the AVM.

\section{Discussion}

In patients with feeder aneurysms associated with cerebellar AVMs, ruptured aneurysm should be the first target of treatment by surgical or endovascular techniques. Aneurysm clipping and AVM resection in a single surgical session is a widely used strategy to address aneurysms associated with $\mathrm{AVMs}^{1,6,7}$. Coiling of the aneurysm in the acute phase followed by surgical resection of the AVM or radiosurgery is another treatment option ${ }^{2.3 .5}$. In case 1 , although the patient was referred to us in the subacute stage we thought that the ruptured aneurysm, which still carried a high risk of rebleeding, should be treated as soon as possible.

We chose to treat our patients by embolizing the aneurysm and AVM simultaneously with NBCA, as we believe that simultaneous embolization of the 2 lesions is the least invasive treatment. NBCA was the only material that could achieve simultaneous embolization of the 2 lesions. To our knowledge, this treatment option has not been detailed in the literature. Our method is much less invasive than surgical intervention and carries less risk of rebleeding from the residual $\mathrm{AVM}^{4}$.

In case 1 , we embolized the aneurysm from inside. Although the injected glue filled the aneurysm, we speculate that residual inflow due to precautionary heparinization resulted in rebleeding, which was controlled by spontaneous thrombosis of the aneurysm after heparin neutralization. Such rebleeding would have occurred even if we had treated with coils, as coil embolization of aneurysms also requires heparinization and antiplatelet therapy.

In case 2 , a small cerebellar infarct was a treatment complication. It would also have occurred in other treatment options. The ruptured aneurysm was fusiform, and intra-aneurysmal trapping with coils or surgical trapping of the aneurysm would have resulted in infarction of the same territory. Because the severity of such complications depends on the distance between the aneurysm and the AVM, our treatment is appropriate only when the aneurysm is close to the AVM. If the aneurysm is located far proximal to the nidus, a large infarct may develop and result in morbidity or mortality. Although we encountered minor complications, we believe that they were inevitable and that the treatments we performed were successful. 


\section{Conclusion}

Ruptured feeder aneurysms associated with AVMs can be treated in a single procedure by means of embolization with NBCA. This less invasive method is a good treatment option in selected cases.

\section{References}

1. Kikuchi K, Kowada M, Yoneda M: Association of arteriovenous malformation and intracranial aneurysm in the posterior fossa. Surg Neurol 1984; 22: 409-502.

2. Ha JK, Choi SK, Kim TS, Rhee BA, Lim YJ: Multimodality treatment for intracranial arteriovenous malformation associated with arterial aneurysm. J Korean Neurosurg Soc 2009; 46: 116-122.

3. Ezura M, Takahashi A, Jokura H, Shirane R, Yoshimoto T: Endovascular treatment of aneurysms associated with cerebral arteriovenous malformations: Experiences after the introduction of Guglielmi detachable coils. J Clin Neurosci 2000; 7 (Suppl. 1): 14-18.

4. Thompson RC, Steinberg GK, Levy RP, Marks MP: The management of patients with arteriovenous malformations and associated intracranial aneurysms. Neurosurgery 1998; 43: 202-211.

5. Lv X, Wu Z, Li Y, et al.: Endovascular treatment of cerebral aneurysms associated with arteriovenous malformations. Eur J Radiol 2011 Apr 12 [Epub ahead of print].

6. Pasacantilli E, Picherri A, Cuidetti G, Santoro A, Delfini R: Surgical treatment of pial cerebellar arteriovenous fistulas with aneurysm of the main feeding artery. Surg Neurol 2006; 65: 90-94.

7. Mabuchi S, Kamiyama H, Abe H: Distal aneurysm of the superior cerebellar artery and posterior inferior cerebellar artery feeding an associated arteriovenous malformation: Case Report. Neurosurgery 1992; 30: 284-287.

(Received, October 30, 2011)

(Accepted, November 30, 2011) 